\title{
CUMULATIVE AUTHOR INDEX (Volume 10)
}

Abukawa, T. 499

Ahlbehrendt, D. 353

Akasaki, I. 537

Akimoto, K. 431, 519

Albano, E. V. 867

Alcock, S. G. 525

Allison, W. 249

AlShamaileh, E. 641

Amano, H. 537

An, B. 763

Aoki, T. 345

Arakawa, H. 305

Arnault, J. C. 127

Asensio, M. C. 981

Atrei, A. 861

Aubel, D. 55

Avila, J. 981

Azizian, S. 585, 895

Baher, S. 13

Bai, B. 331

Bardi, U. 861

Barlam, D. 263

Barnard, A. S. 233

Barnes, C. J. 641

Bartoš, I. 195

Benslim, N. 677

Bernhard, T. 399

Blum, R.-P. 353

Boerma, D. O. 405

Boggild, P. 963

Bolmont, D. 55

Boo, J.-H. $\quad 629,635$

Borsa, D. M. 405

Böttcher, A. 39

Bouchaud, E. 797

Bouznik, V. M. 101

Braga, R. R. 213

Braun, A. 889

Brizuela, G. 661

Brown, I. G 1

Burghaus, U. 39, 7

Burkholder, L. 909

Caillard, J. 81

Calatayud, M. 175

Cattani, M 1, 571, 903

Chen, G. C. 629

Cho, S.-H. 635

Chu, W.-G. 505

Chuang, T. J. 617

Clark, R. G. 415
Conrad, H. 39

Cortigiani, B. 861

Cottam, M. G. 13

Crompton, K. E. 373

Cui, K. 763

Curson, N. J. 415

Dąbrowski, J. 849

Dávila, M. E. 981

de Izarra, C. 81

Derry, T. E. 295

Diani, M. 55

Diouri, J. 55

Dmytruk, A. 283, 289

Dorfman, S. 213, 227

Du Plessis, J. 365

Emoto, T. 431

Fansuri, H. 549

Finlayson, T. R. 373

Fisher, P. 277

Fissel, A. 849

French, D. 549

Fu, X. 325, 381

Fuks, D. 213, 227

Fukui, Y. 467

Furukawa, Y. 543

Gallego, J. M. 405

Gao, J. 317

Gard, F. S. 669

Gartstein, E. 263

Geng, Z. 95

Glander, G. S. 105

Gobal, F. 895

Gonzalez, E. A. 879

Gonzalez, E. 661

Goto, S. 543

Gotoh, Y. 271, 425

Grachev, S. Y. 405

Grey, F. 963

Gu, N. 569

$\mathrm{Gu}$, S. L. $\quad 625$

Hahn, P. H. 163

Hallam, T. 415

Hanatani, K. 271

Hannech, E. B. 677

Hansen, T. M. 963
Hasegawa, S. 963

Hata, A. 431

Hatanaka, Y. 345

Held, G. 487

Hester, J. 513

Higano, M. 337

Hirao, T. 611

Hisanaga, T. 305

Hoadley, J. 241

Hobara, R. 963

Hofmann, K. R. 605

Holst, B. 249

Honda, S.-I. 611

Hong, I.-H. 617

Hongo, S. 467

Honma, K. 499

Horii, S. 431

Howes, P. B. 525

Hsu, Y.-J. 617

$\mathrm{Hu}$, A. 311

Huan, A. C. H. 493

Huang, F. 95

Huang, L. 569

Huang, X. Q. 311

Hughes, A. E. 365

Hung, A. 169

Hwang, C. S. 591

Ichimiya, A. 431

Ikuno, T. 611

Iloukhani, H. 585

Irurzun, I. M. 23

Ishii, H. 257, 505

Ishikawa, T. 543

Ito, A. 721

Ito, M. 543

Itoh, A. 697

Jakovidis, G. $\quad 443,533$

Jamieson, D. 365

Jamieson, I. M. 443

Jamieson, I. 533

Jasen, P. V. 879

Jeong, J. 121

Jiang, Q. 49

Jiang, S. S. 311,317

Jin, T. 283

Johansson, L. 499

Juan, A. $\quad 661,879$

Jung, C.-K. 635 
Kaburagi, M. 189

Kaito, C. 361, 435, 455, 461

Kamada, K. 611

Kanagawa, T. 963

Kang, B. S. 183

Kang, H. J. 183

Kang, M. 189

Kasapoglu, E. $\quad 737$

Kasuya, A. 305

Katayama, M. 611

Katırcioğlu, S.. 577

Kawaguchi, T. 449

Kawanowa, H. 271, 425

Kawata, H. 431, 519

Keeffe, M. 925

Kido, O. 361, 461

Kido, Y. 389

Kim, H. J. 591

Kim, I. G. 207

Kim, T.-K. 635

Kimura, Y. 361, 455

King, D. A. 23

Kirchner, C. 373

Kitada, M. 473

Klauser, R. 617

Klemradt, U. 373

Kondrikov, N. B. 101

Kono, S. 499

Krupski, A. $\quad 65,843$

Kubler, L. 55

Kulkarni, G. U. 87

Kuryavy, V. G. 101

Kurzina, I. 861

Lahtinen, J. 641

Lamoudi, N. 677

Lang, M. 117

Lapeyre, G. J. 925

Lau, D. C. 277

Lay, G. L. 981

Leckey, R. 669

Lee, B. C. 207

Lee, J. I. 207

Lee, M.-N. 635

Lee, S.-B. $\quad 629,635$

Lerotholi, T. J. 487

Li, J.-Q. 691

Liao, J. 569

Lim, S. 121, 685

Lin, H. 283

Liu, K. 381

Liu, Y. M. 311

Long, J. M. 365

Lu, D. Q. 625

Ma, S. 831

Ma, U. 95

MacLaren, D. A. 249
Maehara, Y. 425

Mak, D. K. 649

Makau, N. W. 295

Makhloufi, B. 677

Matsuda, I. 963

Matsukawa, H. 449

Matsushita, T. 543

McCulloch, D. G. 365

Melo, L. L 1, 571, 903

Menzel, D. 487

Mguig, B. 175

Minot, C. 175

Miranda, R. 405

Mochizuki, T. 543

Mogilyanski, D. 263

Mola, E. E. 23

Monetti, R. 867

Monteiro, O. R 1

Morgan, M. 513

Morgenstern, M. 933

Mortimer, E. C. 241

Moshfegh, A. Z. 745

Mróz, S. 65, 843

Mudie, S. 513

Müller, B. H. 605

Munakata, M. 499

Mundim, K. C. 213

Mundim, K. 227

Nagashima, S. 337

Nagel, O. A. 879

Naitoh, M. 473

Nakamura, M. 543

Niehus, H. 353

Nihei, Y. $\quad$ 257, 505

Ning, H. 95

Nishigaki, S. 473

Nishitani, R. 305

No, S. Y. 591

Nozoye, H. 917

Oberbeck, L. 415

Oh, S. K. 183

Ohashi, H. 543

Ohata, T. 543

Ohkura, S. 611

Okazawa, T. 389

Oshima, C. $\quad 697,721$

Otsuka, I. 337

Oura, K. 611

Ovechko, V. 289

Owari, M. 257, 505

Papageorgopoulos, C. A. 73

Paterson, P. J. K. 365

Pavlov, K. 513, 533

Peng, R. W. 311

Petersen, C. L. 963
Petrakov, A. 533

Pfandzelter, R. 117, 399

Pham, G. H. 549

Pistonesi, C. 661

Plessis, J. D. 241

Puisto, S. R. 487

Punegov, V. 533

Qiu, F. 311

Radny, M. W. 201

Rao, G. R. 917

Rawle, J. L. 525

Read, M. N. 481

Riley, D. J. 249

Riley, J. D. 669

Rokuta, E. 697, 721

Rotenberg, E. 925

Rout, B. 365

Rovida, G. 861

Russo, S. P. 233, 241

Sainio, J. 641

Saito, A. 543

Saito, Y. $\quad 361,435,455,461$

Sakamoto, Y. 467

Sakata, O. 543

Saltas, V. 73

Salvadori, M. C 1, 571, 903

Sari, H. 737

Sasaki, H. 305

Sato, T. 361, 435

Schattke, W. 195

Schmidt, W. G. 163, 221

Schofield, S. R. 415

Seino, K. 221

Seitz, M. 373

Sha, H. 317

Shchitovskaya, E. V. 101

Shen, B. 625

Shen, H. 569

Shevlyuga, V. 861

Shi, H. 201

Shi, Y. 625

Shimizu, R. 505

Shimomura, M. 499

Shimura, T. 543

Shin, J. C. 591

Shiraki, I. 963

Shiraki, S. 257, 505

Shoji, F. 473

Simmons, M. Y. 415

Simon, L. 55

Singh, A. 443

Sirghi, L. 345

Smith, P. V. 201

Snook, I. K. 169, 233

Souda, R. 271 
Spencer, M. J. S. 169

Stacchiola, D. 909

Stonham, A. 365

Strasser, T. 195

Sugiyama, H. 431, 519

Sumitani, K. 519, 543

Sumiyama, K. 305

Sun, C. Q. 1009

Sun, Y. Y. 493

Suzuki, H. 361, 455

Sökmen, I. 737

Tabuchi, M. 513, 537

Tajima, A. 721

Tajiri, H. 431, 519, 543

Takahashi, S. 543, 611

Takahashi, T. $\quad 431,519,543$

Takahasi, M. 543

Takeda, Y. 513, 537

Takeshita, K. 543

Takeuchi, J. 467

Tamine, M. 727

Tamura, K. 257, 505

Tan, W. S. 317

Tanabe, F. 963

Tanaka, T. 697, 721

Tanigaki, T. 361, 455

Tanikawa, T. 963

Tateishi, Y. 305

Teichert, C. 837

Toh, S. K. 365

Tong, S. Y. 831, 925

Toyama, N. 473

Tysoe, W. T. 909
Ueba, H. $\quad 771$

Ueno, H. 361

Urano, T. 467

Urazgil'din, I. 117

Uruga, T. 543

Usher, B. F. 669

Vallée, O. 81

Vasilyeva, M. S. 101

Vaz, A. R. 571

Vicente, J. L. 23

Vickers, R. E. M. 277

Vijayalakshmi, S. 87

Vinod, C. P. 87

Wang, C. R. 605

Wang, F. 625

Wang, X. 325, 381

Wang, Y. 95

Wee, A. T. S. 493

Wei, D.-H. 617

Wibawanta, S. 549

Widstrand, S. M. 499

Winter, H. 117, 399

Wu, H. S. $\quad 831,925$

Wu, X. S. 317

Xie, H. 751

Xiu, X. Q. 625

Xu, L. 569

Xu, M. 837

$\mathrm{Xu}$, S. H. $\quad 831,925$

Xu, Y.-J. 691
Yamashita, K. 697

Yang, J.-R. 331

Yang, L. 317

Yaoita, M. 337

Yarovsky, I. 169

Yazawa, T. 283

Yin, G.-C. 617

Yong, K. 121

Yong, K. 685

Yoshimura, M. 467

Yu, Y. 381

Yu, Z. X. 831

Yuan, C. 763

Zakeri, K. H. 745

Zhang, X. 519

Zhang, D. K. 549

Zhang, P.-C. 331

Zhang, R. 625

Zhang, T.-J. 763

Zhang, X. 431

Zhang, Y.-F. 691

Zhang, Y. 569

Zhao, D. S. 49

Zhao, M. 49

Zhao, Z. 325, 381

Zheng, Y. D. 625

Zhou, H. W. 751

Zhou, S.-Q. 331

Zhu, J. 95

Zou, J.-S. 331 


\section{CUMULATIVE AUTHOR INDEX (Volume 11)}

Abdallah, M. S. 427

Abdel-Rahman, M. A. 427

Abouelhassan, S. 83, 199

Adhikari, S. 553, 559, 585

Adhikary, S. 553, 559, 585

Ahonen, M. 457

Arabski, J. 563

Atuchin, V. V. 191

Azizi, A. 563

Badawi, E. A. 427

Badawi, E. 167,451

Bartoš, I. 447

Belhadi, M. 87, 99, 321

Belykh, S. F. 391

Bhavsar, S. R. 443

Bilić, A. 185

Borca, C. N. 531

Boumrar, H. 155

Bozzolo, G. 235, 591

Brongersma, H. H. 341

Burghaus, U. 521

Canto, G. 77

Canzian, A. 235

Carabineiro, S. A. C. 385

Cattani, M. 223, 283, 463

Ch. Argirusis 419

Chadli, R. 321

Chai, J. C. 217

Chen, G. 15

Chen, Q. 305

Chen, X. 15

Cheng, J. 27

Cheng, X. 211

Chou, W.-C. 229

Creemers, C. 341

Dai, C. Y. 433

Dai, X. Q. 1

Dang, H. 111

Dashti, A. 137

Dashti, M. 33

de Groot, F. M. F. 385

Deng, D. 599

Desai, C. F. 443

Dew, S. K. 391

Dinia, A. 563

Dong, S. 205

Dowben, P. A. 531

Du, W. 379, 491
Duan, C.-G. 531

El-Mansy, M. K. 199

Elmansy, M. 83

Fan, D. B. 27

Farías, D. 591

Farias, R. J. C. 223

Foulias, S. D. 419

Ganesan, V. 265

Gasmi, A. 337

Geng, Y. L. 71, 379, 491

Gesari, S. B. 145

Gunes, M. 49

Güneş, M. 403

Guo, L. X. 367

Guo, L. 291, 497

Gupta, V. K. 41

Han, J. 211, 503

Hannech, E. B. 337

Hardt, D. E. 217

Hasegawa, N. 553

He, Z. 7

Helfensteyn, S. 341

Hirsimäki, M. 457

Huang, S. Y. 515

Hush, N. S. 185

Iqbal, K. 117

Irurzun, I. M. 57

Jeong, H.-K. 531

Jiang, B. L. 367

Jiang, D. 205

Jiang, S. 433

Jimbo, T. $553,559,569,577,585$

Joshi, K. P. 41

Juan, A. 145

Junell, P. 457

Kamaratos, M. 419

Kasapoglu, E. 49, 403, 411

Kato, S. 553

Kesler, V. G. 191

Kh. Zakeri 137

Khan, K. M. 117

Khater, A. 87, 99

Khirman, M. A. 259

King, D. A. 57

Kjeldgaard, L. 385

Komesu, T. 531
Kumar, U. 451

Lacharme, J.-P. 21

Lam, Y. C. 217

Lamoudi, N. 337

Lei, T. Q. 367

Lei, T. 291, 497

Li, D. Y. 173

Li, H. 211

Li, J.-S. 509

Li, Q. 111,503

Li, T. B. 71

Li, W. 173

Li, Y.-R. 509

Lim, G. C. 305

Lin, J. S. 229

Liu, H. Y. 379, 491

Liu, J. 531

Liu, R. 291, 497

Lu, M.-W. 331

Luyten, J. 341

Mao, L. 111

Maschke, K. 87

Mei, W. N. 531

Meng, H. 599

Miao, Y. 7

Miranda, R. 591

Mishra, R. K. 271, 541, 607

Misra, K. D. 271, 541, 607

Mokutani, H. 553

Mola, E. E. 57

Mosca, H. O. 235

Moshfegh, A. Z. 33

Mukhopadhyay, P. K. 451

Murukeshan, V. M. 217, 259

Ng, V. M. 515

Nieuwenhuys, B. E. 385

Omer, A. M. M. $553,559,585$

Ozturk, E. 297

Pagura, C. 391

Palmstrøm, C. J. 531

Pan, D. 503

Pan, Y. 433

Pandey, R. P. 607

Park, B. 179, 373

Peng, C. 485,599

Petukhov, A. G. 531

Pokrovsky, L. D. 191 
Pronsato, M. E. 145

Qin, X. 205

Rafil, O. 155

Rafti, M. 57

Rathore, M. K. 41

Rathore, V. 41

Read, M. N. 447

Reimers, J. R. 185

Romero, M. T. 77

Rubensson, J.-E. 385

Rusop, M. 553, 559, 569, 577, 585

Ryu, Y. 373

Salman, F. 83, 199

Salvadori, M. C. 223, 283, 463

Sari, H. 49, 297, 403, 411

Schultz, B. D. 531

Schurmans, M. 341

Sheha, E. 83, 199

Shrivastava, S. B. 41

Sinha, O. P. 265

Soga, T. 553, 559, 569, 577, 585

Sökmen, I. 49, 297, 403, 411

Song, S. 485, 599

Soni, P. H. 443

Srivastava, P. C. 265

Stepanova, M. 391

Sun, D. L. $\quad 71,379,491$
Takeuchi, N. 77

Tamine, M. 155

Tan, J. L. 259, 305

Tiwari, R. P. 541, 607

Tolstogouzov, A. 391

Tong, S. Y. 1

Tran, D. V. 217

Uchida, H. 553, 559, 585

Umeno, M. 553, 559, 569, 577, 585

Valden, M. 457

Vaz, A. R. 223

Vázquez de Parga, A. L. 591

Vicente, J. L. 57

Vlachos, D. 419

Voronkova, V. I. 191

Wang, B. 15

Wang, G. 15

Wang, H. 27

Wang, J. 503, 521

Wang, X. C. 305

Wang, X. Q. 71, 379, 491

Wang, Y. L. 491

Wang, Y. M. 367

Wang, Z. 211, 503

Wu, H. S. 1

Xie, M. H. 1
Xu, D. $\quad 71,379,491$

Xu, M. 515

$\mathrm{Xu}$, S. H. 1

Xu, S. Y. 515

Yan, H. 27

Yang, C. 211, 503, 509

Yang, H. 211

Yang, J. 363

Yanovskii, V. K. 191

Yesilgül, U. 411

Yin, W.-G. 531

Yong, K. 179, 373

Yoshimura, M. 27

Yu, S. 7

Yu, W.-F. 509

Yu. Maklakova, N. 191

Zaïbi, M.-A. 21

Zhang, G. H. 71, 379, 491

Zhang, S.-M. 311

Zhang, T. 363

Zhang, X. 7

Zhang, Y. C. 27

Zhang, Z. 111

Zhao, J. 15

Zheng, H. Y. 217, 259, 305

Zhou, Y. C. 433 


\section{CUMULATIVE AUTHOR INDEX \\ (Volume 12)}

Abdallah, M. S. $\quad$ 197, 203, 463, 519, 545

Abdel-Latif, R. M. 197

Abdel-Rahman, M. A. 197, 203, 463, 519, 545

Abdulrazaq, O. A. 299, 515

Adachi, M. 19

Adhikari, S. 13

Adhikary, S. 13

Avilés, F. 101

Azizi, A. 391

Badawi, E. A. 1, 197, 203, 463, 493, 519, 545

Bao, Q. 539, 597, 773, 819

Belash, V. P. 713

Boumrar, H. 85

Cattani, M. 221, 675

Ceh, O. 101, 289

Chai, J. C. 351

Chen, C. 401, 505, 539, 555, 597, $773,781,819$

Chen, C. Z. 41, 123, 161, 209, 369, 443, 741

Chen, G. 279, 417

Chen, J.-X. 753

Chen, L. 539, 597

Chen, L. X. 741

Chen, Y. 709

Chua, S. J. 651

Corona, J. E. 289

Cui, Y.-J. 753

Dai, S. $\quad 59,749$

Dai, X.-Q. 483

Dai, Z. 397

Ding, P. 75

Dinia, A. 391

Dong, Q. 555, 781

Dong, S. M. 549

Drofenik, M. 97, 239

Du, Z. 59

Fang, Z. 355, 489

Feng, T. 733

Fenineche, N. 391

Filardo Bassalo, J. M. 221

Fort, T. 457

Foulias, S. D. $\quad 721,787$

Gao, L. $\quad 355,489$

Gao, S. 433
Ge, H.-L. 753

Geng, Y. L. 413

Gesari, S. B. 227

Ghodsi, F. E. $\quad 425,793$

Gu, S. 409

Hamzah, E. 631

Han, J. 409

Hashim, A. H. 631

He, P. 749

Huang, S. 397

Huo, Q. H. $\quad$ 41, 161, 209, 443, 741

Irigoyen, B. L. 227

Ismail, R. A. 299, 515

Ji, Q. 369, 555, 781

Jia, F. 489

Jiang, D. 799

Jiang, D. L. 549

Jiang, X. 59

Jimbo, T. $\quad 13,19,27,35,167$, 173, 185, 325, 333, 343, 579, 587, 605, 681, 691, 697

Ju, W.-W. 483

Juan, A. 227

Kamaratos, M. 721

Kan, Y. 561

Kang, H. 355

Kasapoglu, E. 155

Kawano, H. 107

Khan, T. 759

Kinugawa, T. 27

Klimova, I. N. 713

Lam, Y. C. 351

Lan, J. 75

Lei, T. $\quad 539,819$

Lei, T. Q. 41, 123, 161, 209, 443

Li, Q. $\quad 733$

Li, Y. 59

Lim, G. C. 651

Liu, D. 709

Liu, J. 539

Liu, P. 619

Liu, S. 7, 319, 499, 727

Liu, X. 733

Liu, W. 561, 569, 573, 651

Long, J. 397

Lu, M.-W. 67

Lu, Y. 355
Ma, G. 409

Ma, J. 773

Mafakheri, M. 793

Maldonado, R. D. 289

Maqbool, M. 759, 767

Masumdar, E. U. 703

Mishra, R. K. 47

Misra, A. P. 47

Misra, K. D. 47

Mitrokhin, Yu. S. 713

Mohd Toff, M. R. Hj. 631

Mominuzzaman, S. M. 35

Monteiro, P. J. M. 233

Mubarak, A. 631

Natarajan, B. 645

Novinrooz, A. 793

Ng, F. L. 651

Oliva, A. I. 101,289

Omer, A. M. M. 13

Park, B. 745

Perdikis, A. 787

Qin, X. H. 549

Rafil, O. 85

Ramakrishnan, V. 645

Ramamurthy, S. 645

Riveros, H. G. 289

Rusop, M. 13, 19, 27, 35, 167, 173, 185, 325, 333, 343, 579, 587, 605, 681, 691, 697

Ryou, J. 233

Sahari, A. 391

Salvadori, M. C. $\quad 221,675$

Sari, H. 155

Schmerber, G. 391

Sharon, M. 579, 587, 691

Shi, J.-J. 433

Shirata, T. 681

Soga, T. $\quad 13,19,27,35,167$, 173, 185, 325, 333, 343, 579, 587, 605, 681, 691, 697

Sökmen, I. 155

Song, S. 457

Sun, Y. 397

Tak, Y. 215

Tamine, M. 85

Tan, S. $\quad 75,799$ 
Tan, X. 611

Tian, Y. S. 41, 123, 161, 209, 369, 443, 741

Tigrine, R. 85

Tong, S. Y. 483

Tripathi, A. K. 47

Uchida, H. 13

Uma, K. 605, 697

Umeno, M. 13, 35, 579, 587, 691

Uskoković, V. 97, 239

Vasu, V. 645

Vlachos, D. $\quad 721,787$

Wang, D. 59, 401, 505, 539, 555, $773,781,819$

Wang, D. Y. 41, 123, 161, 209, 369,443

Wang, X. 733

Wang, X. C. 651

Wang, X. Q. 413

Wang, Z. 409

Wu, L.-N. 753

Wu, X. 489
Xiao, B. L. 549

Xiao, H. Y. 809

Xie, M. H. 483

Xu, D. 413

$\mathrm{Xu}$, J. $\quad 561,569,573,733$

Xu, L. 809

Xu, S. 397

$\mathrm{Xu}, \mathrm{Z} . \quad 569$

Yahya, K. Z. 299, 515

Yakovkin, I. N. 449

Yang, C. 409

Yang, D. 355, 409

Yang, H. 355

Yang, J. 355, 749

Yang, Y. 409

Yao, L. 773

Ye, Q.-L. 753

Yi, D.-Q. 499

Yi, Y. 799

Yin, M. 355, 489

Yong, K. 215, 745

Yu, G. W. 413

Yu, S.-J. 753

Yu, Z.-M. 499
Zhang, G. H. 413

Zhang, J. 733

Zhang, J. Q. 727

Zhang, L. 433, 819

Zhang, M. 749

Zhang, N. 709

Zhang, S. $\quad 115,145,359,379,523$, 623

Zhang, T. 355,489

Zhang, W. 7

Zhang, X. 59

Zhang, X. P. 279, 417

Zhang, Y. 457

Zhang, Z. 749

Zhao, Y. 401, 505, 597

Zheng, H. Y. 351

Zheng, J. 355, 489

Zheng, X. J. 611

Zhou, Y. C. 611

Zhou, Z. 75, 799

Zhu, H. 351

Zou, S. 733

Zu, X. T. 809 


\section{CUMULATIVE AUTHOR INDEX \\ (Volume 13)}

Abdel-Rahman, M. A. 485

Abdelhamed, M. O. 485

Abdullah, S. 1, 7, 573, 577, 585,593

Abo-Elsoud, M. 773

Adhikari, S. $\quad$ 577, 585, 593, 789

Adhikary, S. 789

Ali, M. 763

Anwar, M. 457

Ashour, A. $\quad 69,87$

Badawi, E. $\quad 69,485$

Bao, Q. 93, 103, 451, 655

Bao, R. 509, 645

Bastrukov, S. 81

Borstel, G. 149

Brack, N. 319

Braun, W. 155

Brizuela, G. 387

Canto, G. 495

Cao, N. 423

Chen, C. $\quad 35,63,93,103,451$, 503, 509, 545, 551, 645, 655, 687,737

Chen, G. 613

Chen, J.-X. 779

Chen, L. B. 535

Chen, L. $\quad 545,551$

Chen, S. 669

Chen, T. 123

Chen, Y.-M. 423

Chen, Y. W. 123, 833

Chen, Z. Y. 57

Cheng, M. F. 519

Cheng, Y.-Y. 265

Cheng, Z. 313

Chihaya, H. 201

Chung, T.-W. 279

Cortie, M. B. 297

Dai, C.-A. 265

Dai, X. 429

Daimon, H. 191, 197, 209

Damiani, D. 387

Dawson, P. 273

Ding, E. 819

Dong, Q. 35, 63, 503, 509

Dong, Z. C. 143

Edamoto, K. $\quad$ 185, 227

Eftekhari, A. 703, 753, 809
Eglitis, R. I. 149

El-Sayed, N. Z. 69

Feng, P. X. 711, 847

Ford, M. J. 297

Fu, P. 429

Gard, F. S. 215

Ge, H.-L. 779

Geng, Y. L. 111, 607

Ghauri, I. M. 457

Gong, J. 369

$\mathrm{Gu}$, S. 13

Guo, F. Z. 209

Guo, P. S. 123

Guo, W. F. 111

Guo, X. L. 143

Guo, X. P. 57

Hamzah, E. $\quad$ 413, 621, 763

Han, J. 13

Hashem, H. A. 439

Hattori, K. 191, 209

He, P. 51

Hirose, K. 179, 309

Honda, M. 197

Hou, J. G. 143

Hou, X. Q. 111

Huang, S. M. 833

Huang, S. Y. 833

Huang, S. 123

Huang, W. 251

Ishikawa, H. 329

Ismail, H. 773

Jayavel, R. 803

Ji, Q. 35

Jia, Y. 841

Jian, X. G. 733

Jiang, F. 13

Jiang, Z. 471

Jimbo, T. 1, 7, 577, 585, 593

Jin, C. X. 833

Juan, A. 387

Kamaratos, M. 681

Kamiko, M. 201

Kang, E.-T. 251

Kang, E. T. 313

Kanno, I. 167

Kasapoglu, E. 397, 747
Kashyap, S. 221

Kataoka, K. 191, 209

Kato, M. 185, 227

Kato, Y. 209

Kawaguchi, T. 235

Kazemzad, M. 703, 753

Kera, S. 259

Keyanpour-Rad, M. 703, 753

Kim, J. 567

Kobayashi, E. 259

Kobayashi, N. 179, 309

Kojima, I. 201

Kou, Y. 733

Kushvaha, S. S. $\quad 241,287$

Lai, P.-Y. 81

Lee, K. Y. 635

Lei, T. 451, 655

Lei, T.-Q. $\quad 545,551$

Li, D. J. 173

Li, H. Y. 847

Li, J. 841

Li, M.-S. 423

Li, W. 819

Li, X. D. 123, 833

Li, Y. 613

Li, Y. R. 27

Li, Z. 403

Lian, J. 471

Liesegang, J. 319

Lim, D. 795

Liu, J. $\quad$ 451, 841

Liu, P. 785

Liu, Q. X. 173

Liu, S. 661

Liu, W. 403

Liu, X. 471

Liu, X. J. 607

Liu, Y. 815

Liu, Z. Y. 733

Long, J. 123

Lu, M. 599

Lü, Y.-P. 423

Luan, Y. 429

Luo, X. D. 519

Ma, G. 13

Ma, J. 93, 103, 687

Ma, Q.-S. 423

Ma, X. G. 825

Maldonado, R. D. 557

Markovic, N. M. 697 
Mase, K. 259

Masens, C. 297

Matsui, F. 197, 209

Matsukawa, H. 235

Matsushita, T. 209

Mori, E. E. 681

Mubarak, A. 413, 621

Muhamad, M. R. 573

Mun, B. S. 697

Murthy, L. C. S. 357

Murugakoothan, P. 803

Nakano, S. 329

Natarajan, B. 351

Neoh, K. G. 251, 313

Ni, C. 123

Nie, J. L. 45

Nishimura, T. 191

Noh, D. Y. 167

Ogiso, H. 329

Oh, S. 201

Okudaira, K. K. 259

Oliva, A. I. 557

Omer, A. M. M. 577, 585, 593

Ordejón, P. 495

Osovskii, V. D. 375

Ozaki, K. 329

Ozaki, T. 309

Ozawa, K. 185, 227

Park, Y. W. 635

Pei, Z. 369

Peng, J.-W. 251

Petrova, N. V. 375

Pigram, P. J. 319

Ploog, K. H. 155

Podder, J. 1, 7

Prince, K. 215

Prissanaroon, W. 319

Ptushinskii, Yu. G. 375

Qi, B. $\quad 103,545,645$

Que, W. X. 123

Ramakrishnan, V. 351

Ramamurthy, S. 351

Rao, K. N. 221, 357

Rathnakumari, M. 803

Refat, M. S. 439

Riley, J. D. 215

Riveros, H. G. 557

Ross, Jr., P. N. 697

Rossi, M. 697

Rusop, M. 1, 7, 573, 577, 585, 593

Ryou, J. 345
Sankar, R. 803

Sankara Narayanan, T. S. N. 635

Santoso, E. G. 795

Sari, H. 397, 747

Sato, T. 185,227

Sellai, A. 273

Seo, S. H. 167

Shah, S. 345

Shamala, K. S. 357

Shi, H. 149

Shi, Y. C. 847

Shi, Z. L. 313

Shimamoto, Y. 191

Siddheswaran, R. 803

Siddiqi, S. A. 457

Simonetti, S. 387

Sobhy, M. S. $\quad 479,773$

Soga, T. 1, 7, 577, 585, 593

Sökmen, I. 397, 747

Song, M. 687

Song, X. 117

Song, Y. 841

Song, Y. Y. 607

Stamenkovic, V. 697

Sugimoto, W. 201

Sui, J.-L. 423

Sun, C. 369

Sun, Z. 123, 833

Sureshkumar, P. 803

Suzuki, T. 167

Tang, C.-Q. $\quad 825$

Teh, K. M. 795

Tian, Y. 551

Toff, M. R. M. 413, 621, 763

Tsui, T.-A. 265

Uchida, H. 789

Ueno, N. 259

Umeno, M. $\quad$ 577, 585, 593, 789

Vasu, V. 351

Wakayama, Y. 143

Wan, S. 795

Wang, B. 117

Wang, D. 35, 63, 103, 451, 503, 655, 669, 687, 737

Wang, H. 369, 737

Wang, J. 815

Wang, J. Y. 733

Wang, L. L. 123

Wang, M. X. 173

Wang, Q. 329

Wang, Q.-X. 423

Wang, S. 841
Wang, X. 503, 613, 841

Wang, X. Q. 111, 607

Wang, X.-S. 241, 287

Wang, X. Z. 123

Wang, Z. 759

Wang, Z. G. 45, 365

Wang, Z. Y. 607

Wasa, K. 167

Watanabe, M. 697

Wu, H. 279

Wu, H. S. 815

Xiao, H. Y. 45

Xiao, W. 241, 287

Xie, M. H. 815

Xu, D. 111,607

$\mathrm{Xu}, \mathrm{H} . \quad 13,369$

Xu, J. 201

Xu, M. 287

Xu, M.-J. 241

$\mathrm{Xu}$, S. 123

Xue, S. 759

Yakovkin, I. N. 375

Yamamoto, R. 201

Yan, H. 117, 369

Yan, Q. L. 733

Yan, Z. 241, 287

Yang, B. Q. 847

Yang, C. 13, 27

Yang, D. 13, 51

Yang, J. 51

Yang, J. H. 519

Yang, M. 403

Yang, X.-H. 825

Yao, L. 93, 103

Yao, S. 489

Yao, Y. 489

Yesilgul, U. 747

Yi, Y. 27

Yong, K. 567

Yu, G. W. 607

Yu, H. 509, 645

Yu, M. 117, 369

Yu, S. 669

Yu, S.-J. 779

Zhang, G. H. 111

Zhang, H. 471

Zhang, H. B. 111, 607

Zhang, J. J. 173

Zhang, J. Q. 661

Zhang, L. 75, 489, 545, 551, 645,655

Zhang, M. 51

Zhang, Q. 57 
Zhang, S. 17,127

Zhang, T. H. 519

Zhang, X. 613

Zhang, Y.-J. 779

Zhang, Z. J. 123
Zhao, X. 841

Zhao, Y. 451,687

Zheng, H. Y. 795

Zhong, M. 403

Zhou, Y. 117
Zhu, H. B. 833

Zhu, X. 313

Zu, X. T. $\quad 45,365$ 